\title{
The Genera of Collembola (Insecta) in Puerto Rico: Keys, Diagnoses, and General Comments ${ }^{1}$
}

\author{
José A. Mari Mutt ${ }^{2}$
}

\begin{abstract}
Keys are presented to the families and genera of Collembola of Puerto Rico. Generic diagnoses also are presented. A previously unrecorded family and genera are added to the known Collembola of Puerto Rico.
\end{abstract}

\section{INTRODUCTION}

The present work was the result of one year of collecting throughout Puerto Rico. Most collecting was by the author, but others contributed material. The principal collection was by Berlese funnels. Hand collecting provided additional material.

All Puerto Rican genera of Collembola are not represented herein. Detailed study of ecological niches such as the epigeon, caves, littoral zones, etc. doubtlessly will furnish additional records to those included here.

The keys and diagnoses presented have been designed for the nonspecialist. These keys are not as simple to use as the pictorial keys by Scott (40), but the illustrations and characters used will make them useful to anyone with a general knowledge of entomology and some experience in morphology of Collembola.

The classical five-family classification was adopted for the sake of simplicity. More elaborate systems, such as that proposed by Salmon (34), was not justified by the nature and coverage of the present work.

The synonymy presented in the diagnoses corresponds to the first and last synonyms that have appeared for the given genus. For complete synonymy until 1964, refer to Salmon (34). The author has detailed synonyms which appeared since 1964.

${ }^{1}$ Manuscript submitted to Editorial Board September 30, 1974.

${ }^{2}$ Graduate Student, Department of Entomology, University of Illinois, Urbana-Champaign 61801. The author expresses appreciation to Dr. David L. Wray, Department of Entomology, North Carolina State University, Raleigh, N.C.; Dr. Kenneth Christiansen, Department of Biology, Grinnell College, Grinnell, Iowa; and Dr. Peter F. Bellinger. Department of Biology, California State University, Northridge, California, for checking the determinations; to Silverio Medina Gaud and Dr. Jenaro Maldonado Capriles, for the additional insect material they contributed, to Dr. Maldonado for suggesting the theme and for his encouragement throughout investigation conducted under his guidance in the Department of Biology, University of Puerto Rico, Mayagüez, Puerto Rico. 
When several genera share many characteristics in common, a fairly detailed diagnostic description for one of the genera has been made. The diagnoses for the related genera include only differences with respect to the first.

Ant. 1, and Abd. 1, etc., means first, second, etc., antennal or abdominal segment, respectively.

\section{REVIEW OF LITERATURE}

In 1948 Wolcott (50) cited seven genera of Collembola for Puerto Rico: Lepidocyrtus, Entomobrya, Cyphoderus, Salina, Campylothorax, Xenylla, and Hypogastrura (Achorutes of Wolcott). Wray (51) added nine additional genera: Pseudosinella, Dicranocentrus, Dicranocentropha, Dicranocentruga, Portachorutes, Brachystomella, Sphyrotheca, Folsomia, and Isotomiella. These papers, together with that of Ramos (30) in which Lepidocyrtinus is recorded from Mona Island, and Wray's (52) record of Drepanocyrtus, constitute all that had been published on the Collembola of Puerto Rico. Eleven new records of genera are added herein to the collembolan fauna of Puerto Rico. These are marked with an asterisk (*) in the keys.

In 1953 Wray (51) cited Isotoma minor from Puerto Rico. This species was transferred to the genus Isotomiella. Since no species of Isotoma was collected in Puerto Rico, the record was eliminated.

For preparation of the keys some decisions must be explained to avoid misunderstanding.

Wray (51) divided Dicranocentroides Imms into two subgenera: Dicranocentroides s. str. and Dicranocentropha. Salmon (34) raised the latter subgenus to generic status. Wray (53) agreed with Salmon, therefore, Salmon's change was adopted.

Dicranocentrus Schott was divided by Wray (51) into the subgenera Dicranocentrus s. str. and Dicranocentrella. Salmon (34) eliminated the latter subgenus synonymyzing it with Dicranocentrus. Wray used Dicranocentrella as a genus. Again, Salmon was followed.

Rapoport (31) cited Dicranocentroides fasciculatus Imms and Dicranocentrus gracilis Schött for Puerto Rico, apparently unaware of Salmon's changes. Dicranocentroides fasciculatus has not been found in Puerto Rico and neither has Dicranocentrus gracilis. The species present here is Dicranocentrus marias Wray.

Rapoport (32) considered Portachorutes Wray (51), as "dubious" and did not include it in his list of the endemic Neartic genera. However, following Salmon (34), the author considered the genus cited to hold generic status. 


\section{GENERAL COMMENTS}

Some authors regard Entomobryoides as a genus. Others consider it to be a subgenus of Entomobrya as originally proposed. They differ chiefly in that Entomobryoides has a double row of smooth hairs (simple setae) on the inner face of the tibiotarsus, which Entomobrya s. str. lacks. Following Maynard (26), the author considered Entomobryoides to be a subgenus of Entomobrya. Material has been collected in Puerto Rico belonging to both subgenera.

Some authors regard Ballistrura as a subgenus of Proisotoma. Others regard it as a genus. They differ in that Proisotoma s. str. has the dentes dorsally slightly crenulated and ventrally clothed with a few setae. Ballistrura lacks both these characters. The author followed Maynard (26), Gisin (19), and Christiansen (13) in regarding Ballistrura as a subgenus. The material collected in Puerto Rico belongs to the latter subgenus.

Similarly, Sphaeridia is regarded by some authors as a subgenus of Sminthurides, and to hold generic ranking by others. They differ chiefly in that Sphaeridia lacks the tibiotarsal organ. The author followed Maynard (26), and Ginsin (19) and regarded Sphaeridia as a subgenus. The material collected in Puerto Rico belongs to the subgenus Sphaeridia.

Many of the characters used in differentiating genera, and particularly subgenera in the Sminthuridae, are structures which are usually difficult to see or interpret. For this reason, the key to this family is not based on these characters. They have, nevertheless, been mentioned in the diagnoses so that the interested reader may get acquainted with their names and locations.

The generic collembolan fauna of Puerto Rico has not been compared with that of the Caribbean or Neotropical region because the fauna of these areas is too poorly known. For example, as far as the author knows, only one genus has been reported thus far from the Dominican Republic where forty or more may occur.

\section{KEY TO THE WORLD FAMILIES OF COLLEMBOLA}

1. Body globular; thoracic and abdominal segments fused (suborder Symphypleona) (Plate I, fig. 2) ................. Sminthuridae Body elongate; segmentation distinct, except sometimes in Abd, 4-6 or Abd. 5-6 (Plate I, figs. 1, 2) (suborder Arthropleona) ........... 2

2. Prothorax well developed, similar to meso- and metathorax, clothed with setae dorsally (Plate I, figs. 8, 9) . . . . . . . . . . . 3 

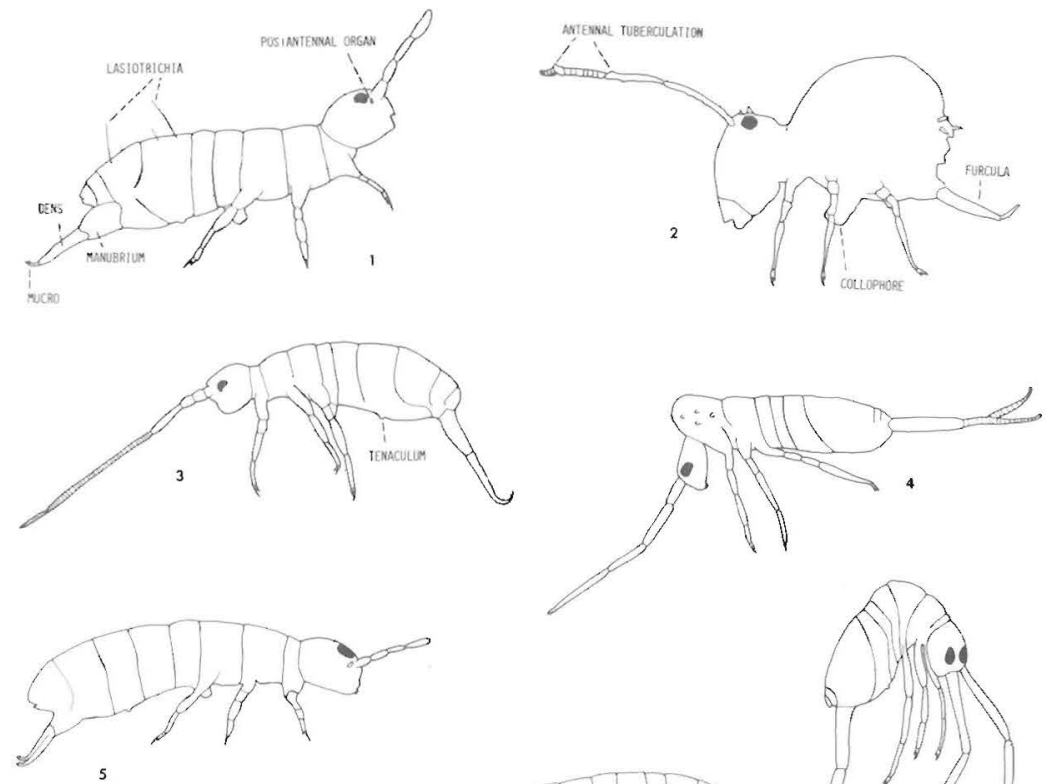

5
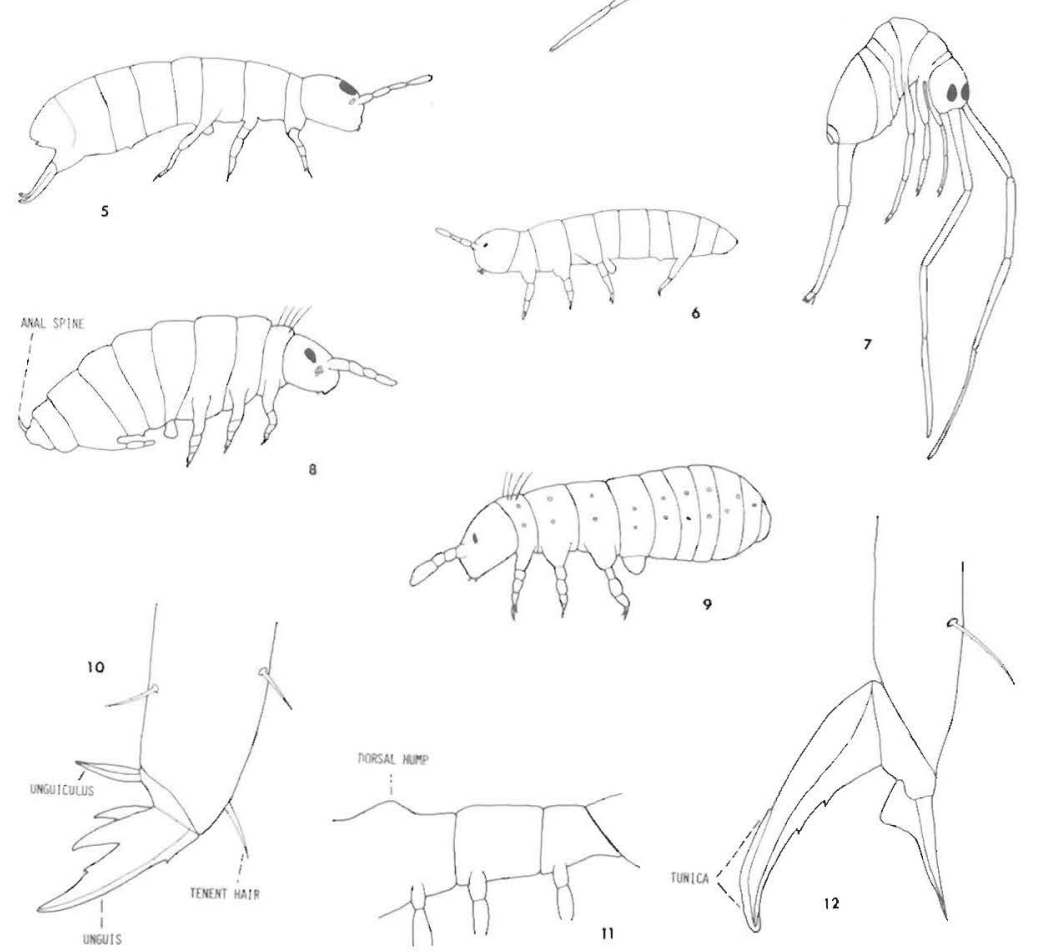

Plate I: Fig. 1. Isotomurus sp.; fig. 2. Ptenothrix sp.; fig. 3. Dicranocentrus sp.; fig. r. Lepidocyrtus sp.; fig. 5. Cryptopygus sp.; fig. 6. Folsomides sp.; fig. 7. Dicranocentropha sp.; fig. 8. Hypogastrura sp.; fig. 9. Onychiurus sp.; fig. 10. Pseudosinella sp., claw and tenent hair; fig. 11. Campylothorax sp., part of body showing dorsal hump on metathorax: fig. 12. Dicyrtomina sp., claw with tunica. 

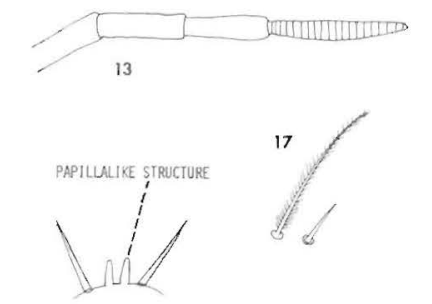

16
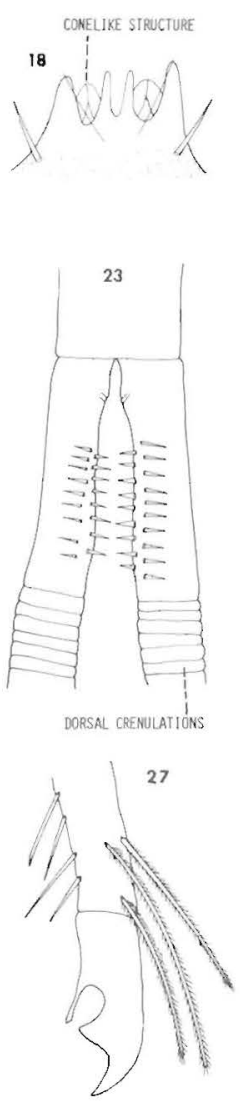

24
14

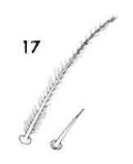

19

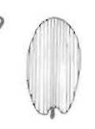

22
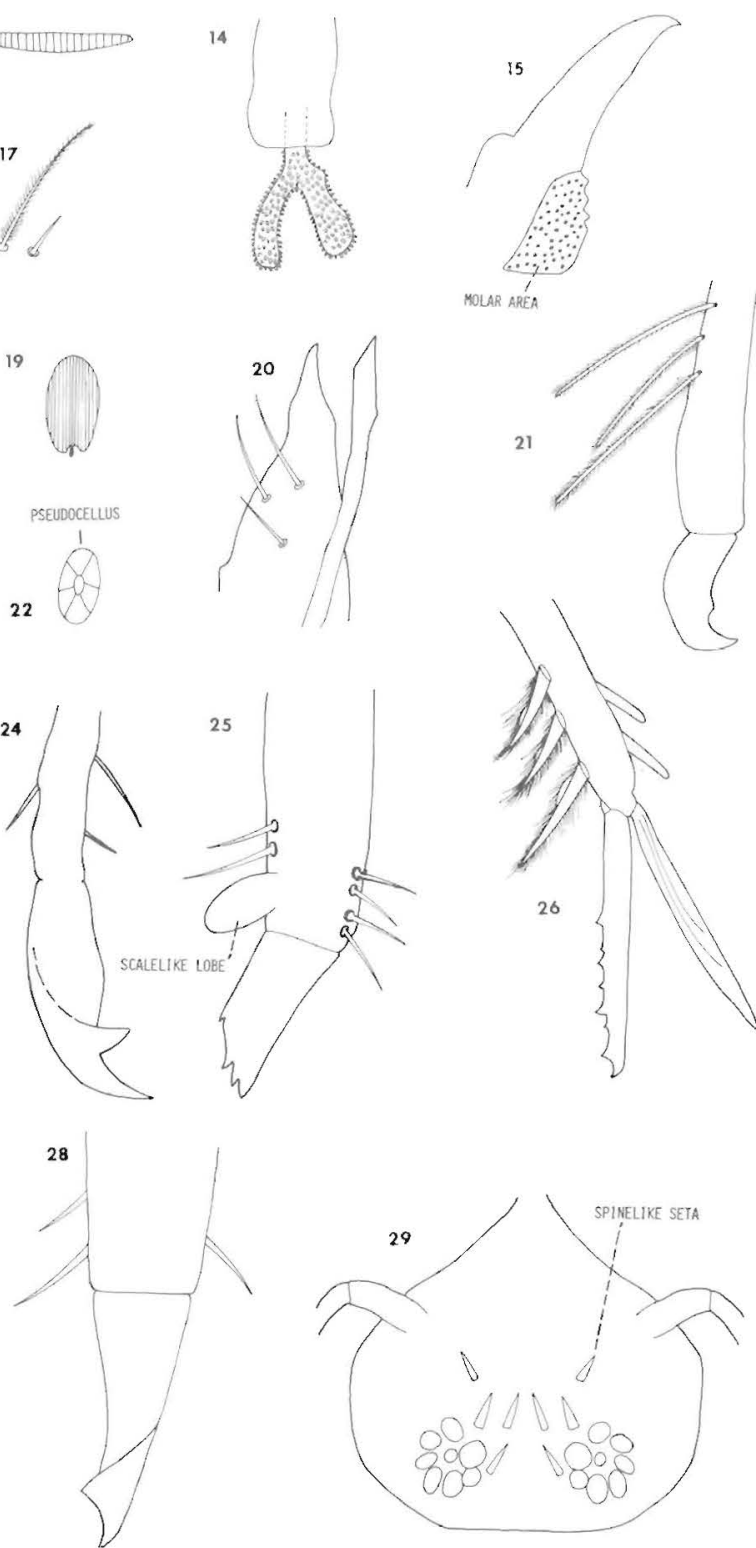

Plate II: Fig. 13. Lepidocyrtinus sp., antenna with annulations; fig. 14. Sphyrotheca sp., exsertile filaments of collophore; fig. 15. Hypogastrura sp., mandible with molar area; fig. 16. Hypogastrura sp., Ant. 3 sense organ with papilla-like structures and setae; fig. 17. Isotomurus sp., lasiotrichia and normal setae; fig. 18. Onychiurus sp., Ant. 3 sense organ with papilla-like, conelike structures, and setae; fig. 19. Lepidocyrtus sp., scale of body; fig. 20. Pseudachorutes sp., mandible and maxilla; fig. 21. Drepanocyrtus sp., mucro; fig. 22. Onychiurus sp., pseudocellus; fig. 23. Dicranocentrus sp., dorsal view of end of manubrium and proximal half of dentes; fig. 24. Cryptopygus sp., mucro; fig. 25. Salina sp., mucro; fig. 26. Cyphoderus sp., mucro; fig. 27. Lepidocyrtus sp, mucro; fig. 28. Proisotoma sp., mucro; fig. 29. Sphyrotheca sp., dorsal view of head with spinelike setae. 
Prothorax reduced, partially or completely hidden by mesothorax, not clothed dorsally (Plate I, figs. 3, 4) . . . . . . . . . . . . . . . 4

3. Integument with regularly distributed pores (pseudocelli) (Plate I, fig. 9, Plate II, fig. 22); Ant. 3 sense organ composed of conelike and papilla-like structures (Plate II, fig. 18); eyes absent; generally unpigmented

Onychiuridae Pseudocelli always absent; Ant. 3 sense organ only with papilla-like structures (Plate II, fig. 16); eyes usually present; usually pigmented Poduridae

4. Abd. 4 dorsally at least 1.5 times Abd. 3 (usually many times longer); furcula long and completely developed, reaching forward to the collophore; scales often present; (Plate II, fig. 19); postantennal organ usually absent (Plate I, figs. 4, 7) ............... Entomobryidae Abd. 4 shorter than Abd. 3, subequal, or when longer, slightly so; furcula reaching or not to the collophore; scales always absent; postantennal organ usually present (Plate, I, figs. 1, 5) .... Isotomidae

\section{FAMILY ENTOMOBRYIDAE TOMOSVARY, 1882 (44) SENSU LATO}

Key to the Genera of Entomobryidae of Puerto Rico

1. Dens crenulated dorsally (Plate I, fig. 4, Plate II, fig. 23); furcula tapering considerably along its length (Plate I, fig. 3) ..........2 Dens smooth dorsally; furcula tapering little (Plate I, fig. 7) . . . . 7

2. Antennae 6-segmented; dentes bearing spines (Plate II, fig. 23); Abd. 4 dorsally not over 1.5 times Abd. 3 (Plate I, fig. 3) ... Dicranocentrus Antennae 4-segmented; dentes without spines; Abd. 4 dorsally over 2.5 times Abd. 3 (Plate I, fig. 4) . . . . . . . . . . . . . . . . 3

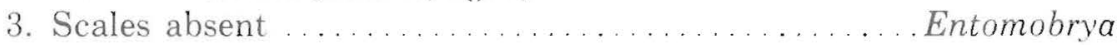
Scales present

4. Eyes less than eight to each side; claws with two basal winglike teeth which are variable in size (Plate I, fig. 10) .......... Pseudosinella Eyes $8-8$; claws without winglike teeth $\ldots \ldots \ldots \ldots \ldots \ldots \ldots$

5. Ant. 4 annulated (Plate II, fig. 13) ............. Lepidocyrtinus Ant. 4 not annulated . . . . . . . . . . . . . . . . . 6

6. Mucrones with two teeth and a basal spine (Plate II, fig. 27). Mucrones falcate (Plate II, fig. 21) ............. Drepanocyrtus

7. Dentes dorsally with large, conspicuous, ciliated scales (Plate II, fig. 26); mucrones long, at least one-fifth the length of the dentes Cyphoderus

Dentes not as above; mucrones many times smaller than the

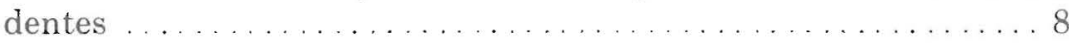

8. Manubrium longer than dentes; Ant. 4 annulated, in some speci- 
mens slightly Dicranocentruga

Dentes longer than manubrium; Ant. 4 not annulated ..........9

9. Scales absent; dentes with a characteristic scalelike lobe at apex (Plate II, fig. 25); teeth of mucrones no more than four ....... Salina Scales present, dentes without apical scalelike lobe; mucrones with five or more teeth ............................ 10

10. Thorax normal in shape (Plate I, fig. 7); eyes 8-8 . Dicranocentropha Metathorax with a dorsal hump (Plate I, fig. 11); eyes 6-6 Campylothorax

\section{Genus Lepidocyrtus Bourlet}

Lepidocyrtus Bourlet, 1839: 391 (11).

Paidium Koch, 1840: 356 (21).

Ascocyrtus Yosii, 1962: 9 (55).

Antennae 4-segmented, not annulated; eyes 8-8 on dark patches; postantennal organ absent; scales present, conspicuous or transparent; mesonotum dorsally obscuring pronotum, often projecting over the head; tenent hairs present, well developed; Abd. 4 dorsally over 2.5 times Abd. 3 ; dentes scaled ventrally, crenulated dorsally; mucrones with two teeth and a basal spine.

\section{Genus Lepidocyrtinus Börner}

Lepidocyrtinus Börner, 1903: 154 (8).

Differs from Lepidocyrtus in that Ant. 4 is annulated and the mucrones are falciform.

Genus Drepanocyrtus Handschin

Drepanocyrtus Handschin, 1924: 17 (20).

Differs from Lepidocyrtus in that the mucrones are falciform, and from Lepidocyrtinus because Ant. 4 is not annulated.

\section{Genus Pseudosinella Schaeffer}

Tullbergia Lie-Pettersen, 1896: 16 (22).

Pseudosinella Schaeffer, 1897: 38 (36).

Pettersenia Scherbakow, 1898: 61 (37).

Differs from Lepidocyrtus in that the eyes are less than eight to each side; tenent hairs often reduced in size; claws bear two winglike teeth.

\section{Genus Entomobrya Rondani SENSU LATO}

Entomobrya Rondani, 1861: 40 (33).

Choreutes Burmeister, 1838: 449 (12).

Parentomobrya Dahl, 1912: 424 (14). 
Contrary to Lepidocyrtus, Entomobrya lacks scales and the mesonotum is not prominent.

\section{Genus Dicranocentrus Schött}

Dicranocentrus Schött, 1893: 21 (38).

Dicranocentrella Wray, 1953: 140 (51).

Antennae 6-segmented, segments 5-6 annulated; ocelli 8-8 on dark patches; postantennal organ absent; mesonotum not prominent; scales present; Abd. 4 not over 1.5 times Abd. 3; dentes scaled ventrally, dorsally crenulated, and with longitudinal rows of spines; mucrones with two teeth and a basal spine.

\section{Genus Cyphoderus Nicolet}

Cyphoderus Nicolet, 1841: 381 (29).

Cyphodurus Nicolet, 1841: 384 (29).

Beckia Lubbock, 1870: 279 (25).

Antennae 4-segmented; eyes absent; postantennal organ absent; Abd. 4 much longer than Abd. 3; claws with large winglike teeth; dens smooth dorsally, with conspicuous fringed scales larger near the apex, also scaled ventrally; mucrones long, from one-half to one-fifth the length of dentes, with a variable number of teeth.

\section{Genus Salina MacGillivray}

Salina MacGillivray, 1894: 107 (28).

Cremastocephalus Schött, 1896: 175 (39).

Antennae 4-segmented, not annulated, longer than body; eyes 8-8, on dark patches; postantennal organ absent; mesonotum not prominent; tenent hairs present, well developed; Abd. 4 many times longer than Abd. 3; furcula long, tapering little throughout its length; dentes not crenulated, longer than manubrium, with an apical scalelike lobe at apex, not bearing spines; mucrones with up to four teeth; scales absent.

\section{Genus Dicranocentruga Wray}

Dicranocentruga Wray, 1953: 141 (51).

Differing from Salina, Dicranocentruga has: Antennae shorter than body, fourth segment annulated; ocelli 6-6; scales present; manubrium longer than dentes; dentes with spines and a long apical process not scalelike; mucrones with five teeth.

\section{Genus Dicranocentropha Wray}

Dicranocentropha Wray, 1953: 144 (51).

The characters that separate this genus from Salina are: Scales 
present; dentes with spines, apical scalelike lobe absent; mucrones with five to seven teeth.

\section{Genus Campylothorax Schött}

Campylothorax Schött, 1893: 18 (38).

This genus differs from Salina in: Ocelli 6-6; scales present; dorsal hump present on metathorax; dentes with spines, without apical scalelike lobe; mucrones with six teeth.

The author has reason to believe that this determination might be in error. Dicranocentrapha (not described) when Wolcott's paper appeared, could have been taken easily for Campylothorax. It has been impossible to trace the material cited by Wolcott. Also, Campylothorax has not appeared in these collections, while Dicranocentropha is abundant everywhere in Puerto Rico.

\section{FAMILY ISOTOMIDAE BÖRNER, 1913 (10)}

\section{Key to The Genera of Isotomidae of Puerto Rico}

1. Furcula long, reaching forward to the collophore; dentes crenulated dorsally (Plate I, fig. 1) ............................. . . Furcula short, not reaching the collophore; dentes crenulated or not (Plate I, fig. 5)

2. Eyes absent; postantennal organ absent; Abd. 5-6 fused; lasiotrichia (bothriotrichia of other authors) absent .............. Isotomiella Eyes present; postantennal organ present; Abd. 5-6 separated; lasiotrichia present (Plate I, fig. 1, Plate II, fig. 17) ....... Isotomurus*

3. All abdominal segments separated; body elongated, tubelike (Plate I, fig. 6) Folsomides* Abd. 4-6 or Abd, 5-6 partially or completely fused; body not as above

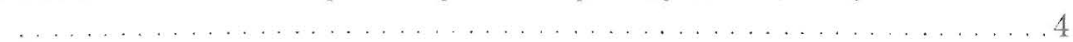

4. Abd. 4-6 fused into a single mass ................... Folsomia Abd. 4-6 not fused; Abd, 5-6 partially or completely fused . . . . . . . 5

5. Mucrodens joint distinct (Plate II, fig. 28) ............ Proisotoma* Mucrodens joint indistinct (Plate II, fig. 24) ...........Cryptopygus*

\section{Genus Isotomurus Börner}

Isotomurus Börner, 1906: 71 (9).

Antennae 4-segmented, not annulated; eyes 8-8 on dark patches; postantennal organ present; tenent hairs absent; Abd 5-6 not fused; lasiotrichia present; furcula long, reaching the collophore; dentes dorsally crenulated, without spines; mucrones with four teeth. 
Genus Isotomiella Bagnall

Isotoma Bourlet, 1839: 401 (11) in part.

Isotomiella Bagnall, 1939: 95 (2).

Differing from Isotomurus, Isotomiella has: Eyes, postantennal organ, and lasiotrichia absent; Abd. 5-6 fused; mucronal teeth less than four.

\section{Genus Proisotoma Börner SENSU LATO}

Proisotoma Börner, 1901: 172 (7).

Scutisotoma Bagnall, 1948: 535 (3).

Folsomidiella Bagnall, 1949: 59 (4).

Antennae 4-segmented, not annulated; eyes 8-8, on dark patches; postantennal organ present; Abd. 4 subequal or slightly longer than Abd. 3; Abd. 5-6 partially fused; lasiotrichia absent; furcula not reaching the collophore; dentes with or without ventral setae, crenulations present or absent; mucrones with 2-3 teeth; mucrodens joint distinct.

Genus Cryptopygus Willem

Cryptopygus Willem, 1902: 11 (48).

Isotomina Börner, 1903: 140 (8).

Hemisotoma Bagnall, 1949: 94 (5).

This genus differs from Proisotoma mainly in the following: Abd. 4 subequal to, or shorter than Abd. 3; Abd. 5-6 completely fused; mucrodens joint indistinct.

\section{Genus Folsomia Willem}

Folsomia Willem, 1902: 280 (49).

Litsteria Bagnall, 1949: 60 (4).

Hemisomia Bagnall, 1949: 92 (5).

This genus differs from Proisotoma mainly as follows: Eyes vary in number from 0 to 8-8; Abd. 4-6 fused.

\section{Genus Folsomides Stach}

Folsomides Stach, 1922: 17 (41).

The characters that separate Folsomides from Proisotoma chiefly are: Eyes 5,2, or 1 to each side; body much elongated, tubelike; Abd. 5-6 separated.

FAMILY PODURIDAE LUBBOCK, 1870 (25) SENSU LATO

Key to the Genera of Poduridae of Puerto Rico

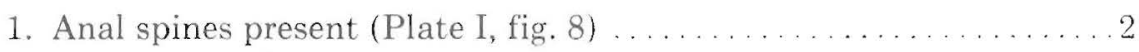

Anal spines absent 
2. Mandibles without a molar area; anal spines $2-15 \ldots \ldots \ldots$. . . Friesea* Mandibles with a well-developed molar area (Plate II, fig. 15); anal

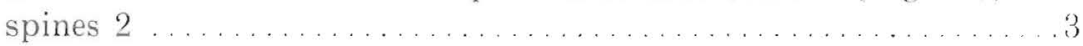

3. Eyes 8-8; unguiculus present; postantennal organ present (Plate I, fig. 8) Hypogastrura

Eyes 5-5; unguiculus absent; postantennal organ absent .... Xenylla

4. Eyes 5-5; postantennal organ absent ............... Portachorutes Eyes 8-8; postantennal organ present ................. 5

5. Mandibles present; maxillae styliform (Plate II, fig. 20) Pseudachorutes* Mandibles absent; maxillae with several teeth Brachystomella

\section{Genus Pseudachorutes Tullberg}

Pseudachorutes Tullberg, 1871: 155 (46).

Brachysius MacGillivray, 1893: 317 (27).

Neoafricella Salmon, 1964: 115 (34).

Antennae 4-segmented, conical, subequal to head in length; eyes 8-8 on dark patches; postantennal organ present, composed of a variable number of tubercles; mouthparts projected forward, like in a cone; mandibles with one or more teeth; maxillae styliform; body plump; integument tuberculate; anal spines absent; furcula short, not reaching the collophore but fully developed; mucro spoonlike.

\section{Genus Portachorutes Wray}

Portachorutes Wray, 1953: 147 (51).

This genus differs from Pseudachorutes chiefly in: eyes 5-5; postantennal organ absent.

\section{Genus Brachystomella Ågren}

Brachystomella Ågren, 1903: 127 (1).

Schoettella Schaeffer, 1896: 175 (35) in part.

Brachygastrura Rapoport, 1962: 443 (31).

Differing from Pseudachorutes, Brachystomella has: mandibles absent; maxillae with several teeth.

\section{Genus Friesea Dalla Torre}

Friesea Dalla Torre, 1895: 14 (15).

Triaena Tullberg, 1871: 155 (46).

Neocolonavis Salmon, 1964: 267 (34).

This genus differs from Pseudachorutes in: Eyes vary from 0 to 8-8; postantennal organ absent; mouthparts not so markedly projecting 
forward; maxillae sickle-shaped; anal spines present, 2-15; furcula very small, rudimentary, well developed, or absent.

\section{Genus Hypogastrura Bourlet}

Podura Linné, 1758: 608 (23) in part.

Hypogastrura Bourlet, 1839: 404 (11).

Cyclograna Yosii, 1960: 263 (54).

Hypogastrura can be readily separated from Pseudachorutes in: Mouthparts not projecting forward; mandibles with one to several teeth, and with a well-developed molar area; maxillae with several teeth; unguiculus present; anal spines present, 2 .

\section{Genus Xenylla Tullberg}

Xenylla Tullberg, 1869: 11 (45).

This genus differs from Pseudachorutes essentially in the same characters as Hypogastrura. It differs from the latter genus in: Eyes 5-5; postantennal organ and unguiculus absent.

\section{FAMILY ONYCHIURIDAE BÖRNER, 1913 (10) SENSU LATO}

Only one genus of this family is known to occur in Puerto Rico: Onychiurus and this is the first record of the family in this country. The material collected in Puerto Rico belongs to the subgenus Onychiurus s. str.

\section{Genus Onychiurus Gervais SENSU LATO}

Lipura Burmeister, 1838: 447 (12).

Onychiurus Gervais, 1841: 372 (18).

Orthonychiurus Stach, 1954: 26 (41).

Body stout; head dorsally as wide or wider than prothorax; antennae 4-segmented, subequal to head in length; Ant. 3 apically with a sensory organ composed of various different structures; postantennal organ present, compound, with many small tubercles; pseudocelli present, regularly distributed throughout head and body; unguiculus present; furcula usually absent, or represented by a rudimentary structure; pigment generally absent.

\section{FAMILY SMINTHURIDAE LUBBOCK, 1862 (24) SENSU LATO}

Key to the Genera of Sminthuridae of Puerto Rico

1. Ant. 4 longer than Ant. 3, no antennal segment with swellings or protuberances along the margin ....................... 
Ant. 4 much smaller than Ant. 3, Ant. 2-3 with swellings or protuberances along the margin (Plate I, fig. 2) ...............

2. Parts of body and top of head with spinelike setae (Plate II, fig. 29); Ant. 4. subsegmented; exsertile filaments of collophore tuberculate (Plate II, fig. 14) .............................. Sphyrotheca Body and head without spinelike setae; Ant. 4 not subsegmented; exsertile filaments of collophore smooth .................... 3

3. Eves 8-8 Sminthurides* Eyes 4-4 Collophora*

4. Ant. 3-4 subsegmented (Plate I, fig. 2); claw without a tunica Ptenothrix* Ant. 4 not subsegmented; claw with a tunica (sometimes barely visible) (Plate I, fig. 12) Dicyrtomina*

\section{Genus Sphyrotheca Börner}

Sphyrotheca Börner, 1906: 183 (9).

Lipothrix Börner, 1906: 183 (9).

Afrosminthurus Delamare Devoutteville and Massoud, 1965; 186 (17).

Ant. 4 subsegmented; eyes 8-8; parts of body and top of head with spinelike setae; body without trace of segmentation; trochanteral organ absent; claw with a tunica; tenent hairs absent; exsertile filaments of collophore tuberculate; mucrones spoonlike; mucronal spine (bristle) absent.

\section{Genus Sminthurides Börner SENSU LATO}

Sminthurides Börner, 1900: 616 (6).

Prosminthurus Willem, 1900: (47).

Jeannenotia Stach, 1956: 10 (43).

Ant. 4 not subsegmented; eyes $8-8$; body or head with spinelike setae; segmentation of body sometimes barely visible as light lines; Ant. 2-3 of male modified into a clasping organ; trochanteral organ absent; tenent hairs absent; mucronal bristle absent; tibiotarsal organ present or absent; mucrones without lamellae.

\section{Genus Collophora Richards}

Collophora Richards, in Delamare Deboutteville and Massoud 1964: 34 (16)

This genus differs from Sminthurides mainly as follows: Eyes 4-4; trochanteral organ present. 


\section{Genus Dicyrtomina Börner}

Dicyrtomina Börner, 1903: 167 (8).

Antennae 4-segmented, not annulated, Ant. 4 much shorter than Ant. 3 , Ant. 2-3 with several swellings or protuberances extending shortly from the margin; eyes 8-8; tenent hairs absent; claw with a tunica; mucrones spoonlike.

\section{Genus Ptenothrix Börner}

Ptenothrix Börner, 1906: 185 (9).

This genus differes from Dicyrtomina chiefly in: Ant. 3-4 subsegmented, claw without a tunica.

\section{RESUMEN}

Se presentan claves para las familias y géneros de colémbolos de Puerto Rico. Se incluye además un diagnóstico para los géneros. Once nuevos géneros y una nueva familia se añaden por primera vez a la lista anterior de colémbolos presentes en Puerto Rico.

\section{LITERATURE CITED}

1. Agren, H., Diagnosen einiger neuen Achorutiden aus Schweden (Vorläufige Mittheilung), Entomol. Tidskr. 24: 126-8, 1903.

2. Bagnall, R. S., Notes on British Collembola, Entomol. Mon. Mag. 75: 901-102, 1939.

3. - Contributions towards a knowledge of the Isotomidae (Collembola), I-VI. Ann. Mag. Natur. Hist. 12(1): 529-41, 1948.

4. - Notes on British Collembola, Entomol. Mon. Mag. 85(1017): 51-61, 1949.

5. - Contributions towards a knowledge of the Isomotidae (Collembola), VII-XV. Ann. Mag. Natur. Hist. 12(2): 82-96, 1949.

6. Börner, C., Vorläufige Mitteilung zur Systematik des Sminthuridae Tullb. insbesondere des Genus Sminthurus Latr., Zool. Anz. 23: 609-18, 1900.

7. - Zur Kenntnis der Apterygotenfauna von Bremen und der Nachbardistrikte Beitrag zur einer Apterygotenfauna Mitteleuropas, Abhandl. Ver. Bremen 17: 1-141, 1901.

8. - Über neue altweltliche Collembolen, nebst Bemerkungen zur Systematik der Isotominen und Entomobryinen, S.B. Ges. Naturforsch. Berlin, 1903: 129-82, 1903.

9. - Das System der Collembolen, nebst Beschreibungen neuer Collembolen des Hamburger Naturhistorischen Museums, Mitt. Nat. His. Hamburg, 23: 147-88, 1906.

10. — Die Familien der Collembolen, Zool. Anz. 41: 315-22, 1913.

11. Bourlet, A., Mémoire sur les Podures, Mém. Soc. Sci. Agr. Lille, 1: 377-417, 1839.

12. Burmeister, H., Collembola, In Handbuch der Entomologie, 2(2); 445-58, 1838.

13. Chritiansen. K., Personal Communication, 1974.

14. Dahl, F., Ueber die Fauna des Plagefenngebietes, Part A: Analytische Übersicht der unter A. genannten Tiere, Beitr. Naturdenkin. Berlin, 3: 341-638., (Collembola: 420-7), 1912.

15. Dalla Torre, K. W., Die Gattungen und Arten der Apterygogenea (Brauer) Sep, 46 Prog. Staats. Gym. Innsbruck: 1-23, 1895.

16. Delamare Deboutteville, C., and Massoud, Z., Collophora remanei n. sp., Collembole 
Symphypléone du Perou et remarque sur sa position systématique, Zool. Anz. 172: 30-36, 1964.

17. — et — Collemboles Symphypléones de l'Angola (Première Note), Diamang (Companhia de deumantes de l'Angola), Publicações Cult. 68: 65-103, 1965.

18. Gervais, P., Designation of type and description of genus Onychiurus, Echo Monde Savant, 8: 372, 1841.

19. Gisin, H., Collembolenfauna Europas, Ed. Mus. Hist. Nat. Genève: 1-312, 1960.

20. Handschin, E., Neue Myrmecophile und Termitophile Collembolenformen aus SudAmerika, N. Beitr. Syst. Insektenk. Berlin, 3: 13-9, 21-8, 1924.

21. Koch, C. L., Poduridae, In Fauna Ratisbonensis, by Herrick-Schaeffer, Fürnrohr. Naturh. Topogr. Regensburg. III: 353-9, 1840.

22. Lie-Pettersen, O. J., Norges Collembola. Fortegnelse over de i Norge hidtil observerede arter, Bergens Mus. Aarbog, No. 8: 1-24, 1896.

23. Linné, C., Systema Naturae (Aptera), ed. 10: 608-9, 1758.

24. Lubbock, J., Notes on the Thysanura, Pt. I: Smynthuridae., Trans. Linn. Soc. 23(3): $429-48,1862$.

25. — Notes on the Thysanura, Pt. 4. Trans. Linn. Soc. 27: 277-97, 1870.

26. Maynard, E., A monograph of the Collembola or springtail insects of New York state, Comstock Publ. Co. Ithaca, New York, pp. 1-339, 1951.

27. MacGillivray, A. D., North American Thysanura, I-IV. Can. Entomol. 25: 127-8, 173, 218-20, 313-8, 1893.

28. - North American Thysanura, V. Can. Entomol. 26: 105-10, 1894.

29. Nicolet, H., Note sur la Desoria saltans, Insecte de la Famille des Podurelles, Bibl. Univ. Genève, 32: 381-7, 1841.

30. Ramos, J. A., The insects of Mona Island, J. Agr. Univ. P.R. 30(1): 1-74, 1946.

31. Rapoport, E. H., Colémbolos de Bahía Blanca (Argentina). IV. Acta Zool. Lilloana 28: 443-455, 1962.

32. - The geographical distribution of Neotropical and Antartic Collembola, Pacif. Ins. Mon. 25: 99-118, 1971.

33. Rondani, C., Entomobrya pro Degeeria Nic., In Rondani-Dipterol, Ital. Prodr. 4: 40. 1861.

34. Salmon, J. T., An index to the Collembola, Bull. Roy. Soc. New Zeal, 7: 1-651, 1964.

35. Schaeffer, C., Die Collembolen der Umbebung von Hamburg und Benachbarter Gebiete, Mitt. Nat. Hist. Mus. Hamburg 13: 149-216, 1896.

36. - Apterygoten, Hamburger Magelhaerische Sammelreise, pp. 1-48, 1897.

37. Scherbakow, A. M., Einige Bemerkungen über Apterygogenea. die bei Kiew 1896-1897 gefunden wurden, Zool. Anz. 21: 57-65, 1898.

38. Schött, H., Beitrage zur Kenntnis der Insektenfauna von Kamerun, I. Collembola, Bih. Suenska. Ak. 19(2): 1-28, 1893.

39. - North American Apterygogenea, Proc. Calif. Acad. Sci. 2(6): 169-96, 1896.

40. Scott, H. G., Collembola: Pictorial keys to the Neartic genera, Ann. Entomol. Soc. Amer. 55(5): 104-13, 1961.

41. Stach, J., Apterygoten aus dem Nord Westlichen Ungarn, Ann. Mus. Nat. Hung. 19: $1-75,1922$.

42. - The Apterygotan fauna of Poland in relation to the world-fauna of this group of insects. Family Onychiuridae, Polska Akad. Nauk. Inst. Zool. 1954: 1-129, 1954.

43. — The Apterygotan fauna of Poland in relation to the world-fauna of this group of insects. Family Sminthuridae. Polska Akad. Nauk. Inst. Zool. 1956: 1-287, 1956.

44. Tomosvary, O., Adatok Hazánk Thysanure-Faunájához, Math. Term. Kozlem. Magyar Akad. 18: 119-130, 1882. 
45. Tullberg, T., Om Skandinaviska Podurider af Underfamiljen Lipurinae, Akad. Afhandl. Uppsala, pp. 1-20, 1869.

46. —_ Fortelkning öfver Svenska Podurider, Öfo. K. Vet.-Akad. Förh 28(1): 143-155, 1871.

47. Willem, V., Recherches sur les Collemboles et les Thysanoures. Mém. Cour. Mém. Sav. étr. Acad. Roy. Belgique, 58: 1-144 (Collembola: p-72), 1900.

48. - Collemboles Recuillés par l'Expédition Antarctique Belge. Resultats du Voyage du S. Y. Belica: 1-19, 1902.

49. - Note préliminaire sur les Collemboles des Grottes de Han et de Rochefort. Ann. Soc. Entomol. Belgique, 46: 275-83, 1902.

50. Wolcott. G. N., The insects of Puerto Rico, J. Agr. Univ. P.R. 32: 1-975, 1948.

51. Wray, D. L., New Collembola from Puerto Rico. J. Agr. Univ. P.R. 37(2); 140-150, 1953.

52. - Some new records of Caribbean Collembola, Bull. Brooklyn Entomol. Soc. 54(3): 67-68, 1959.

53. - Personal Communication, 1974.

54. Yosii, R., Studies on the collembolan genus Hypogastrura. Amer. Midl. Natur 64(2): $257-281,1960$.

55. - On some Collembola of Hindukush, with notes on Isotoma Bourlet and its allies. In Uéno Insect Fauna of Afghanistan and Hindukush. Res. Kyoto Univ. Sci. Exp. Karakoram and Hindukush 1955, 4: 3-42, 1962. 